\title{
Video Transmission over Cognitive Radio TDMA Networks under Collision Errors
}

\author{
Abdelaali CHAOUB \\ Laboratory of Electronic and \\ Communication \\ Mohammadia School of Engineers, \\ Mohammed V-Agdal University \\ Rabat, Morocco
}

\author{
Elhassane IBN ELHAJ \\ Department of Telecommunication \\ National Institute of Posts and \\ Telecommunications \\ Rabat, Morocco \\ IEEE Member
}

\author{
Jamal EL ABBADI \\ Laboratory of Electronic and \\ Communication \\ Mohammadia School of Engineers, \\ Mohammed V-Agdal University \\ Rabat, Morocco
}

\begin{abstract}
Cognitive Radio (CR) networks are emerging as new paradigm of communication and channels sharing in multimedia and wireless networks. In this paper, we address the problem of video transmission over shared $C R$ networks using progressive compression source coding associated to fountain codes. We consider a TDMA-based transmission where many subscribers share the same infrastructure. Each Secondary User (SU) is assigned one time slot where he transmits with a certain probability. The given model allows each SU to transmit opportunistically in the remaining slots. Therefore, packets are not only corrupted by reason of Primary traffic interruptions, but also we consider losses caused by collisions between several SUs due to the Opportunistic Spectrum Sharing. We use a redundancy-based model for link maintenance to compensate for the loss of spectrum resources caused by the primary traffic reclaims. Moreover, we setup up many Secondary User Links to mitigate the collision effects. Numerical simulations are performed to evaluate the proposed approaches in view of the average Goodput. We conduct a stability and performance analysis of the system and we highlight the achieved gains when using our transmission model.
\end{abstract}

Keywords-component; Cognitive Radio network; video transmission; TDMA; progressive compression source coding, LT codes; Collision; Goodput.

\section{INTRODUCTION}

Mobile and multimedia communications services have experienced a great evolution over the last decades. Increasing demand for the frequency spectrum resource makes the radio spectrum more precious. This finding is reinforced by the frequency allocation charts around the world [1]. On the other hand, actual observations of the spectrum occupancy taken on some bands reveal the low and discontinuous usage of the licensed spectrum in time and space [2, 3]. Hence the emergence of the Cognitive Radio [4] as a new paradigm to find strategies for enhancing and sustaining the growth of multimedia and wireless networks with limited spectrum.

The CR concept has been proposed in the objective of improving the spectral resources utilization and management. Cognitive devices are allowed to occupy the spectrum that has been left vacant by licensed users. Therefore, every telecommunication system will be divided into two networks: a primary network called Primary Users (PUs), which owns the spectrum license and has full rights on it, and a secondary network called Secondary Users (SUs), which is allowed to use the primary network's bandwidth in case of PU absence. In order to enable the coexistence of both primary and secondary networks within the same architecture, regulatory authorities [5] aim at exploiting the notions of Negotiated and Opportunistic Spectrum Sharing (OSA) for CR networks.

The OSA [6] is a core technique in Cognitive Radio networks to exploit the temporarily unused spectral resources. Licensed spectrum bands are continuously sensed to detect the unoccupied spectrum hole. From that sensing-derived information, Secondary User Links (SULs) are formed from a composition of multiple subchannels (SCs) currently not in use by licensed users. Subchannels selected to create a SUL should be scattered over multiple PU frequencies. The advantages of this principle are two fold: (1) it limits performance degradation due to the interference caused by primary reappearance; (2) it reduces the number of jammed subchannels once the primary user appears during the lifetime of a SUL.

In summary, the Cognitive Radio solution is introduced as an enabling technology for managing and controlling the frequency spectrum allocation. It has gained considerable maturity during the last years. This emerging approach not only promises great future technological advances and seems to meet many needs of today, but also could be exploited for enhancing a wide range of legacy technologies in particular those frequency-spectrum-based like wireless networks [7].

A wireless network refers to, as its name suggests, a network in which at least two devices could communicate without a wire connection, it is among the largest communication technology worldwide. The explosive growth of wireless services, as internet and multimedia, has increased the need for more quality of service and bandwidth. This standard is completely based on the radio frequency resource and in fact influenced by the scarcity of radio spectrum. That's way, in many works $[8,9,10]$, the Cognitive Radio generates a big interest as a key cost-effective solution for the underutilization of frequency spectrum in wireless communication networks. Cognitive Radio based wireless networks promote the objective of supporting large volumes of customers, very important for operators and industrials. The tricky part is tailoring the legacy wireless services to suit the specificities of the CR context, which makes the problem of 
studying the scalable video transmission over CR networks challenging.

Furthermore, there exist many research efforts on the problem of secondary traffic transmission over Cognitive Radio networks (Fig. 1). In [11], Kushwaha, Xing, Chandramouli and Heffes have studied the transmission of multimedia traffic over CR networks, the primary traffic arrival was modelled as a Poisson process and Luby Transform (LT) code (Fig. 2) [12] has been used as channel correcting code and also for some coordination reasons. They have proposed a QoS metric to order the available subchannels in the decreasing order of their quality to establish the transmission link efficiently. They investigate the spectral efficiency of the selected SUL in terms of successful transmission probability of the required number of packets needed for recovering the original multimedia stream. Unfortunately, this study has not considered the opportunistic aspect of the network (Fig. 5) where many SUs transmit in the same CR network and consequently there is an additional packets loss average due to collision effects which degrades considerably the spectral efficiency of the system. In [13], Cuiran and Chengshu have investigated the successful transmission over Cognitive Radio networks shared by several SUs (Fig. 1) using the TDMA technique. They have assumed a slotted transmission; each SU transmits in his assigned slot and can transmit in the other slots with certain probability. The results have been presented in terms of throughput and energy efficiency. They have considered that the only reception failure reason would be packet collisions due to time sharing. This study has not taken into account the interference effects caused by the primary user appearance. The reception failure depends also on the Primary traffic type and arrival model which affects the reception of the whole transmitted message. In addition, it may happen that the SU does not transmit data in his own slot because there may be no data to transfer, hence the probability that the secondary device transmits in his assigned slot should be, in practice, less than one.

In previous work [14], we have done some contribution on the problem of image transmission over lossy networks using progressive source coding associated to fountain codes where the stream delivery is reinforced by the use of Unequal Error Protection based on the block duplication technique. Currently, our work is addressing the video transmission problem through shared Cognitive Radio networks (Fig. 1). That is, our aim is to develop a compression scheme that allows us to generate multiple levels of quality using multiple layers simultaneously with a network delivery protection model that allows us to deliver subsets of layers to a given population of receivers over unreliable subchannels. So, the same issue has been already treated in [15] by using fountain codes under different subchannel selection policies in a fading environment with the

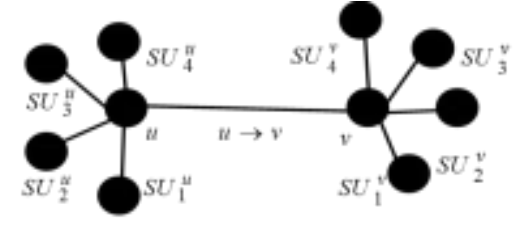

Figure 1. Cognitive Radio network assumption that the primary traffic arrival follows a Poisson process. Herein, we consider the binomial traffics used instead of Poisson where there are a finite number of sources. The given distribution is associated to the general model for link maintenance introduced in [16] (Fig. 3). Some redundancy is added to the secondary applications to combat the interruptions caused by the primary traffic arrival. After sending the message, the used spectrum bands are sensed and the SULs will be restructured in case some packets got lost as a consequence of the PU appearance. We assume a slotted transmission (Fig. 3) and we adopt the TDMA method as a network sharing technique. TDMA allows sharing the same CR infrastructure among multiple subscribers (SUs). The secondary traffic is divided into different time slots (Fig. 5). We consider a centralized scheduler that allocates to each subscriber $S U_{i}$ the time slot $i$ with some probability $q$. Nevertheless, the scheduler tries to maximize the achieved secondary Goodput by using the OSA feature of CR networks, so $S U_{i}$ is allowed to use opportunistically the other slots, let $p$ be the probability that this SU transmits in the remaining slots $j \neq i$ (Fig. 5). The transmission performance on the proposed network model, as the realistic case, is mainly affected by two crucial aspects: (1) interferences caused by the primary traffic arrival leading to more corrupted secondary packets, and (2) packets may collide with one another regarding the fact that each SU attempts to transmit in other slots reserved for other SUs. In our Cognitive Radio network model, collision is defined as the fact that two or more SUs attempt to transmit a packet or many packets across the same Secondary User Link at the same time. Throughout the paper, we develop a system for video transmission based on Joint Source Channel Coding approach. More precisely, we propose to combine a progressive source coder like Set Partitioning in Hierarchical Trees (SPIHT) [17] as the source coding with a fountain code [18] like LT (Luby Transform) codes [12] as the channel coder. The proposed scheme has already shown his benefits and effectiveness in [19]. SPIHT is a high quality source coder based on wavelets, it produces a fully progressive code which means that if the transmission is stopped at any point, a lower bit rate video can still be decompressed and reconstructed. LT code [12] (Fig. 2) is used to cope with packet losses caused by Primary User interference and other channel conditions. Generally, we can also use other fountain codes [18] like raptor codes [20]. It has been shown in [11] that the use of fountain codes kills two birds with one stone. First of all, it avoids the coordination problems between different SCs belonging to the same SUL. Second, it acts as an erasure correcting code. Luby

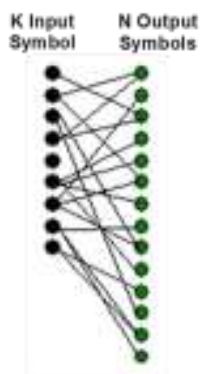

Figure 2. Tanner Graph of LT codes 


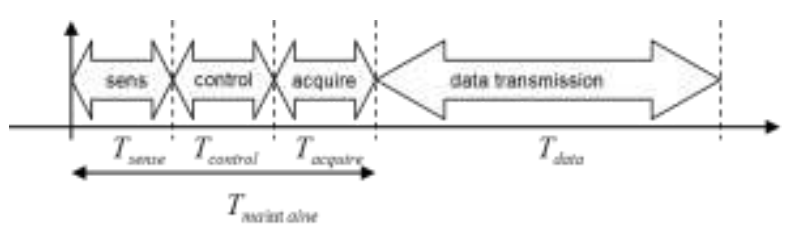

Figure 3. Time frame structure

has used a particularly designed degree distribution for the construction of LT codes called the Robust Soliton Distribution, it has two parameters: $c \succ 0$ and $\delta \in[0,1]$. To reduce the collision effects, we propose to set up many Secondary User Links during the sensing phase (Fig. 3). In [15], we have developed a simple algorithm to establish several SULs having the same efficiency. The formed SULs are pairwise disjoint, which implies that any subchannel belonging to a given SUL can't be reused for constructing other SULs. The existence of many available SULs enables many SUs to transmit in the same time slot without perturbing each other; each SU would be able to transmit in the same time slot and through a specific SUL different from the other subscribers' paths. We assume that collision result in the total communication failure on the chosen SUL, so the occurrence of collisions impedes the performance of the CR network. Moreover, we assume that there is no algorithm to assign to each new secondary user a new secondary user link currently not in use, the existence of such algorithms will reduce the collision probability to the detriment of increasing costs and time delay. We investigate the trade-offs between different system parameter settings and the average Goodput of the developed model. We conclude that, under some parameter settings, the system continue to achieve good performance despite of the presence of primary interferences and secondary collisions. The proposed SULs redundancy-based approach exhibit good results in compensating the performance degradation caused by collisions. We emphasize also the importance of finding a balance that meets quality and Goodput, which means that there is optimal transmission parameters to ensure the expected quality with a given over all Goodput.

The remainder of this paper is organized as follows: In Section 2 we give a brief summary about the Spectrum Pooling concept. We recall the link maintenance model reused in our study. In Section 3 we make use of joint Source Compression and Channel Coding techniques to combine the advantages of both methods. Then, we compute the analytic expression of the achieved Goodput which considers both Primary traffic interruptions and TDMA collisions. In Section 4 we present the numerical results and we show the resulting gains in terms of system Goodput, and finally Section 5 draws our conclusions.

\section{SYSTEM DESCRIPTIONS} study.

Here we introduce some concepts that will be used in our

\section{A. Spectrum Pooling Concept}

The Spectrum Pooling Concept [21] basically consists of selecting several spectral ranges from the primary frequency bands to constitute a common pool. The so called COgnitive Radio for Virtual Unlicensed Spectrum (CORVUS) [22] is based on this approach. The whole frequency spectrum covered by the system is divided into $N$ subchannels each of bandwidth $W=B / N$ where the total available system bandwidth is $B$. The dashed frequency bands in Fig. 4 indicate that de PU is currently active, consequently this frequency band can not be used by any secondary user. The gradient grey color in Fig. 4 shows the vacant subchannels that are selected to construct a Secondary User Link.

Under the single uniform subchannel selection, an SUL should consist of only one subchannel per primary frequency band to ensure a low effect of the PU arrival on a SUL, only one subchannel need to be vacated in case a PU arrives. However, practically, it is expected that in one SUL, more than one subchannel per primary frequency band can be allocated (Fig. 4). Thus, the subchannels within the same frequency band are more likely to be jammed at the same time once the primary user appears, this subchannel selection policy is recommended for cases with available priori knowledge on subchannels state information.

\section{B. Link maintenance model review for primary traffic interruptions}

For the proposed link maintenance model introduced in [16] and as shown in Fig. 3, the frame consists of four parts: a sensing block $T_{\text {sens }}$, a reporting block $T_{\text {control }}$, an acquire block $T_{\text {acquire }}$ and a data transmission block $T_{\text {data }}$.

In the sensing block, all users conduct local spectrum sensing simultaneously. The local sensing results are reported and disseminated between different peers through the Group Control Channel sequentially in the reporting block. Then during the acquire block, new subchannels need to be acquired to compensate for the lost ones. Finally, the next stream is ready to transmission over the cognitive radio network in a delay of $T_{\text {data }}$.

The PU appearance is considered the only reason for a subchannel to be excluded from the SUL. Consequently, the subchannel exclusion probability $p_{x}$ is restricted to the Primary User appearance probability $p_{a}$ :

$$
p_{x}=p_{a} .
$$

In the secondary usage scenario, the SU selects a set of subchannels from the PU bands. The SU is required to vacate the subchannel as soon as the corresponding PU becomes active and claims his spectral resource. Therefore, the secondary user loses some packets on that subchannel. To compensate for that loss, the source packets are encoded with LT codes. Let the secondary user has a message $m$ of $K$ packets to transmit. The LT decoder needs at least $N$ packets in order to

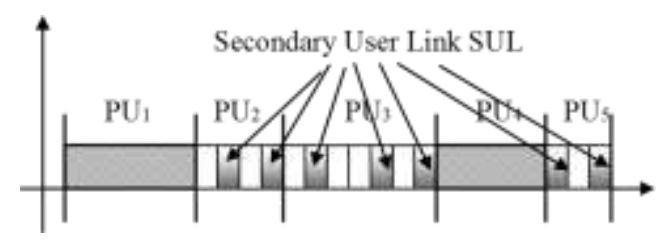

Figure 4. Spectrum Pooling Concept 


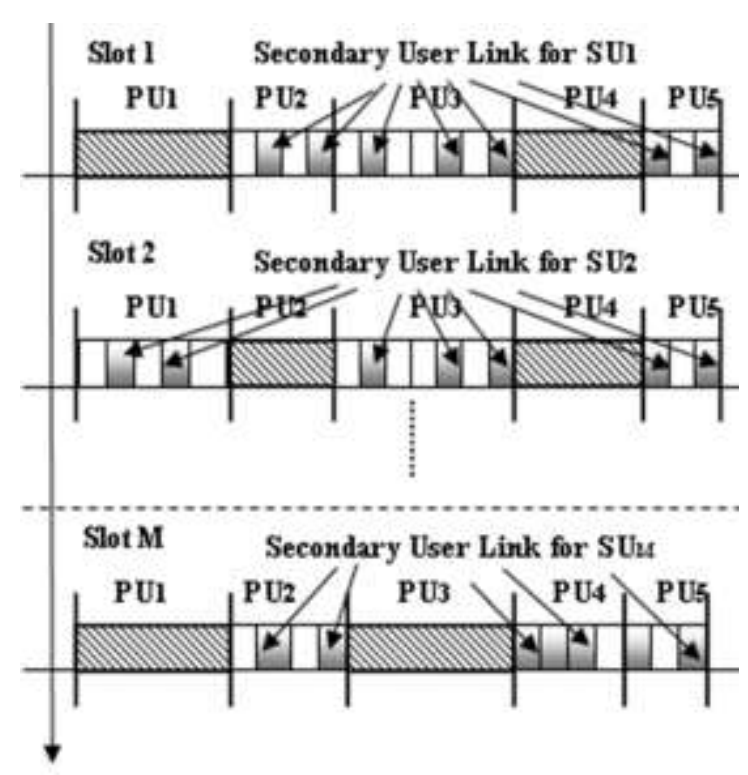

Figure 5. Shared Cognitive Radio network based on TDMA technique. recover the original $K$ packets with probability $1-D E P$. Then in order to compensate for the loss due to PU appearance, we add some redundancy, denoted $X$, which depends on the PU arrival probability $p_{a}$. If the PU arrival is frequent then we need to use high value of $X$. If the PU arrives occasionally then even a small $X$ value will be sufficient. The considered link maintenance model assumes that one packet is transmitted per subchannel. Then, the total number of subchannels used by SU is $S=N+X$. This communication will succeed only if at most $X$ of the subchannels are claimed by their associated licensed users. Hence, the message error probability for secondary users, which take into consideration only the Primary traffic interruptions, is given by:

$$
P_{e r r}=\sum_{i=1}^{N}\left(\begin{array}{c}
N+X \\
X+i
\end{array}\right) p_{a}{ }^{X+i}\left(1-p_{a}\right)^{N-i}
$$

Then the total Goodput can be computed as:

$$
G=\frac{\left(1-P_{\text {err }}\right) \times N \times b_{\text {sc }} \times T_{\text {data }}}{T_{\text {sens }}+T_{\text {control }}+P_{m} \times T_{\text {acquire }}+T_{\text {data }}} .
$$

Where $P_{m}$ is the probability that the SUL has to be restructured and is given by:

$$
P_{m}=1-\left(1-p_{a}\right)^{N+X} .
$$

$b_{s c}$ is the bit rate per subchannel.

\section{PROPOSED NETWORK MODEL}

In this section, we propose a solution to video communication services in cognitive radio context. For this purpose, we give an analytic expression of the Goodput metric which quantifies the QoS requirements of the secondary transmission.

\section{A. General Analysis}

Consider a cognitive radio network where a cognitive source is sending data to a cognitive destination over a spectrum hole unoccupied by licensed users.

In our study, we focus our attention on delay video transmission applications over wireless networks. One or many participants are providing access to video application directly available to a given population of clients with heterogeneous reception bandwidths and quality of service requirements. That is, high quality of service is required and higher data rates must be supported [23, 24, 25, 26].

The video data consists of a group of pictures (GOP). The GOP consists of $K$ packets. We assume that the TDMA frame consists of $M$ slots each of the same time duration $T$.

We introduce the following practical model of TDMA scheduling: Each Secondary user $S U_{i}$ always transmits in his assigned slot $i$ with probability $q$ and transmits with probability $p$ in the remaining time slots $(M-1$ slots $)$.

At the start of every slot $i$, a Secondary User Link is formed by selecting a set of $S$ subchannels from different PU bands of the spectrum pool. Then, $S U_{i}$ starts transmitting his GOP packets over this link during the data duration $T_{\text {data }}$. That is, the sensing part is decoupled from the transmission part. So, we have $T=T_{\text {setup }}+T_{\text {data }}$, where $T_{\text {setup }}=T_{\text {sens }}+T_{\text {control }}+T_{\text {acquire }}$. The probability of PU appearance for any subchannel is given by $p_{a}$. For simplicity of analysis, $p_{a}$ is assumed to be the same for different subchannels.

Despite of the presence of PUs reclaims and SUs concurrency, reliable schemes are required to enable the continuous provision of service for the communication among the secondary network to some satisfying extent. Hence, sophisticated signal processing and coding techniques remains the cornerstone of a successful secondary transmission.

More precisely, in this work we adopt a Joint Source Channel Coding method which is among the most appropriate ways to communicate multimedia content over a lossy packets network. We make use of a progressive encoding system which allows transmitting the coded video as a sequence of layers over CR networks. The use of progressive amounts of redundancy will guarantees a high protection level to the most important data i.e. the base layer of the stream.

In deed, we first form a scalable bit stream $\left(R_{f}\right)_{0 \leq f \leq F}$ by applying SPIHT [17] or any compression scheme on the video [27]. We partition the bit stream source into $F$ layers $\left(L_{f}\right)_{1 \leq f \leq F}$ indexed in order of decreasing importance; we use the fact that a progressive source coder produces an output in which information important to video quality is emitted first. We denote the boundaries of layer $f$ by bits $R_{f-1}$ and $R_{f}$ such that $0=R_{0} \leq R_{1} \leq \ldots \leq R_{F}$. Each layer $L_{f}$ is blocked into $K_{f}$ source blocks. The use of source coding permits to recover the content up to a certain quality commensurate with the number of layers received. 
Let $Q_{f}$ where $1 \leq f \leq F$ be the achieved quality corresponding to the layer $L_{f}$. Regarding the fact that we use a progressing source coding, the reception of the layer $L_{f+1}$ implies the reception of all the subordinate layers $\left(L_{j}\right)_{1 \leq j \leq f}$. Stream $L_{1}$ is the first stream (most important data), and stream $L_{F}$ is the last stream (least important data).

We make use of the LT codes (Fig. 2) to protect the video traffic against PU interferences. We propose to create one fountain code per layer, LT codes is applied on every layer $L_{f}$ where $f \in\{1, \cdots, F\}$. Note $N_{f}$ as the number of LT encoded packets needed to recover the original $K_{f}$ transmitted packets corresponding to the layer $L_{f}$ with probability $1-D E P$. An overhead of $5 \%$ is sufficient in order to reconstruct the data at the receiver, so: $N_{f}=1.05 \times K_{f}$.

Regarding the fact that $N_{f}$ is the minimal amount of encoded packets needed to recover the original video up to the quality $Q_{f}$, any PU interruptions will immediately cause the loss of the layer $f$ and consequently the data of the respective enhancement layers are rendered useless. That is, we add some amount of redundancy, noted $X_{f}$, to overcome the corruption of data packets du to PU arrival.

At a specific time slot, several SUs could be actives and using this slot for transmission or reception at the same time and on the same Secondary User Link. Hence, collisions could occur on the network. Collision errors indicate a serious performance problem on the CR network. We propose a simple way to prevent CR networks from packet collisions [15]. During the sensing phase, many Secondary User Links will be established, such that each active cognitive user will be assigned an SUL different from the others. Therefore, if many SUs' communications coincide at the same time slot, each SU has more chance to take a different SUL and consequently the risk of collision decreases.

As a matter of fact, under a targeted level of quality $Q_{f}$ there are mainly two events that affect the traffic distribution on the selected SUL. A secondary user succeeds his transmission if (1) for the secondary receiver, at least $N_{f}$ packets are received successfully from the set of selected subchannels $S$, and (2) if there is no packet collisions due to the fact that every $S U_{i}$ could transmit opportunistically on other slots not assigned to him. We notice that the last factor is quality independent.

Let $u$ and $v$ be two active secondary users (Fig. 1), the objective is to study the Goodput of the communicatio $u \rightarrow v$ with the sought quality $Q_{f}$. We remember that this transmission is perturbed by the PU reclaims and the collision risks.

Define $P_{e r r, f}^{\prime}$ as the message error probability of the transmission $u \rightarrow v$ with the sought quality $Q_{f}$ (Fig. 1).
In our scheme for secondary use, we define the message error probability as the probability that the active cognitive user $v$ could not reconstruct the GOP sent by $u$ up to the quality $Q_{f}$. In other words, if (1) $X_{f}$ or more subchannels got jammed due to the arrival of PUs, or (2) the transmission $u \rightarrow v$ is subject to collision, the GOP cannot be successfully reconstructed at the receiver with the desired quality $Q_{f}$.

Then, we compute $P_{e r r, f}^{\prime}$ as:

$$
P_{\text {err }, f}^{\prime}=P_{\text {err }, f}+P_{\text {collision }} \text {. }
$$

$P_{e r r, f}$ is the probability that the active cognitive user $v$ fails to receive $N_{f}$ packets over the selected SUL.

$P_{\text {collision }}$ is the probability that there is other SUs trying to access the same SUL as the cognitive user $u$.

B. An Analytical Expression for $P_{\text {err }, f}$

Using the expression (2):

$$
P_{e r r, f}=\sum_{i=1}^{N_{f}}\left(\begin{array}{c}
N_{f}+X_{f} \\
X_{f}+i
\end{array}\right) p_{a}{ }^{X_{f}+i}\left(1-p_{a}\right)^{N_{f}-i} .
$$

\section{An Analytical Expression for $P_{\text {collision }}$}

Let $i$ be the time slot assigned to the active cognitive user $u$ and $D e g_{v}$ defined as the number of neighbors of the active cognitive user $v\left(\left(S U_{i}^{v}\right)_{1 \leq i \leq 4}\right.$ in Fig. 1). We remember that $q$ is the probability that $u$ transmits in his assigned time slot $i$ and $p$ the probability that he transmits in the remaining time slots $j \neq i$ (Fig. 5).

Let $P_{\text {nocollision }}$ be the probability that there is no collisions perturbing the transmission $u \rightarrow v$.

$P_{\text {nocollision }}$ should be derived in the following manner :

For the time slot $i$ :

$$
P_{i, \text { nocollision }}=q(1-p)^{\operatorname{Deg}_{v}} .
$$

For the remaining time slots $j \neq i$, there are two cases: (1) the time slot $j$ coincides with the specific time slot of one of the $v$ neighbors, or (2) there is no user belonging to the $v$ neighbors which owns the time slot $j$.

Hence,

$$
P_{j, \text { nocollision }}=p(1-p)^{\operatorname{Deg}_{v}}+p(1-q)(1-p)^{\operatorname{Deg}_{v}-1} .
$$

We should note that when $q=1$, we obtain the given results in [13].

Using (7) and (8), the average probability of no collisions over the frame and for one Secondary User Link is:

$$
P_{\text {no collision }}=\frac{q(1-p)+(M-1) p(2-p-q)}{M}(1-p)^{\operatorname{Deg}_{v}-1} .
$$


Because the two events are complementary, we have,

$$
P_{\text {collision }}=1-P_{\text {nocollision }} \text {. }
$$

Then, from (9) and (10) we obtain,

$$
P_{\text {collision }}=1-\frac{q(1-p)+(M-1) p(2-p-q)}{M}(1-p)^{\text {Deg }_{v}-1} .
$$

If we consider several structured SULs which are pairwise disjoin as defined in [15], the total average probability of collisions over the available Secondary User Links $N_{S U L}$ is:

$$
P_{\text {collision }}=\left(1-\frac{q(1-p)+(M-1) p(2-p-q)}{M}(1-p)^{\text {Deg }_{v}-1}\right)^{N_{\text {sul }}}
$$

From (5), (6) and (12) $P_{\text {err,f }}^{\prime}$ is completely defined.

We extend the general model of link maintenance introduced in [16] to take the collision aspect caused by the opportunistic transmission into consideration. The total achieved Goodput will be given by:

$$
G_{f}^{\prime}=\frac{\left(1-P_{\text {err }, f}^{\prime}\right) \times N_{f} \times b_{\text {sc }} \times T_{\text {data }}}{T_{\text {sens }}+T_{\text {control }}+P_{m}^{f} \times T_{\text {acquire }}+T_{\text {data }}} .
$$

We recall that $P_{m}^{f}=1-\left(1-p_{a}\right)^{N_{f}+X_{f}} . P_{m}^{f}$ is the probability that the SUL has to be maintained.

\section{Numerical Results}

In this section, we present some numerical results to reinforce the theoretical aspect previously addressed and to outline the achieved gains when using Join Source Channel Coding in Cognitive Radio based wireless networks.

\section{A. General Simulations}

For real video transmission, we consider an MPEG-4 [27] LT encoded video stream with a resolution of $720 \times 576$ pixels and a frame rate of 25 frames/s (DVD quality for example). Our purpose is to study the Goodput average of this transmission on a Cognitive Radio TDMA-based network shared by several Secondary Users.

For the time frame, we suppose that:

$$
T_{\text {sens }}=T_{\text {control }}=T_{\text {acquire }}=T_{\text {data }}=1 \mathrm{~ms}
$$

The Robust Soliton distribution used for the LT coding has as parameters $c=0.1$ and $\delta=0.5$, we consider a decoding error probability of $D E P=0.1 \%$. We assume a BPSK modulation with a code rate of $1 / 2$ which means a bit rate of $b_{s c}=125 \mathrm{kbit} / \mathrm{s}$ per subchannel.

For the given video transmission, we take a data rate of $1.66 \mathrm{Mbit} / \mathrm{s}$, LT codes overhead included. The given data rate represents the maximum achieved Goodput of this transmission. We evaluate the expression (13) by replacing variables with the given values to find the minimal number of packets $N$ needed to ensure this multimedia transmission (we must take $\left.P_{e r r, f}^{\prime}=P_{m}^{f}=0\right)$. Thus, $N_{f}=40$.
Fig. 6 depicts the impact of the number of available Secondary User Links $N_{S U L}$ on the total achieved Goodput plotted against the amount of redundant subchannels $X . D e g_{v}$ and $M$ values has been fixed respectively at 3 and 5 . The estimated traffic average on the assigned slot is $q=90 \%$ and in the remaining slots is about $p=30 \%$. As it is seen and according to what was expected, the Goodput performs good results where increasing the number of available SULs. Indeed, at a specific time slot, if there are many available SULs, it is very unlikely that two Secondary Users transmits over the same SUL and consequently more chance to avoid collisions. It is also interesting to note that there is some $X$ value that maximizes the achieved Goodput. Adding other SCs to the Secondary User Link over this value doesn't give any amelioration; on the contrary, degrades the transmission performance.

For all the following numerical result, the number of available Secondary User Links has been fixed to 20 .

Fig. 7 illustrates the achieved Goodput over Cognitive Radio network shared by several SUs using TDMA techniques plotted against the number of additional subchannels $X$. Here, we study the impact of the probability $q$ on the traffic transmission performance for a fixed value of $p=30 \%$. We have fixed the following settings $D e g_{v}=3$ and $M=5$. Thus, while decreasing the $q$ value, the proposed network model provides better results in terms of Goodput. This is due to the fact that when the SU is increasing the traffic transmission on his assigned slot, eventual collisions on this slot are more likely to happen for a fixed $p$ value.

In Fig. 8, the computed Goodput is given versus the redundancy $X$; simulations were run for several $p$ values for a fixed value of $q=90 \%$. It can be observed that for low $p$ values, the Goodput increases. High traffic performance is attained where approaching $p \approx 0.1$ and the Goodput approaches his maximum value $G_{f}^{\max }=1.66 \mathrm{Mbit} / \mathrm{s}$. It is obvious that where decreasing the traffic among the other slots assigned to the other SUs, we reduce the chance that our SU interferes with the other active cognitive users. There is always an optimal value

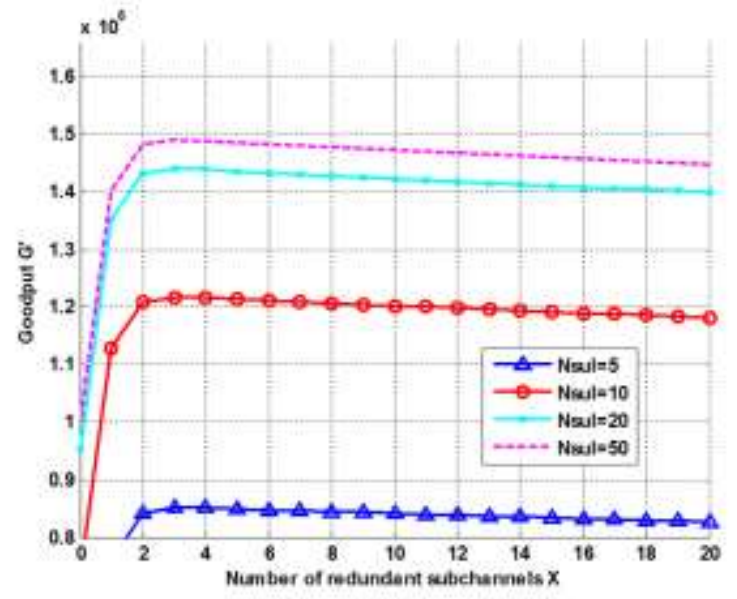

Figure 6. Computed Goodput for different $N_{\text {sal }}$ values 


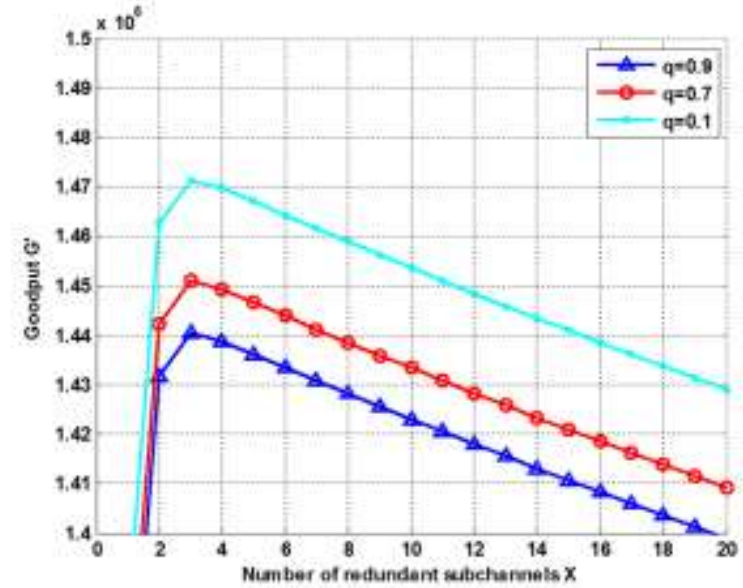

Figure 7. Goodput comparison for different values of the probability $q$ $(p=0.3)$

of the added redundancy that realize a trade-off between the computed Goodput of the system and the added redundancy. On the other hand we notice that there exists an optimal value of the probability $p$ which maximizes the system Goodput $(p \approx 0.1)$.

Fig. 9 represents the Goodput against the redundancy $X$ for several values of $\mathrm{Deg}_{v}$. The slots number has been fixed at 5 and the probabilities $p$ and $q$ has been fixed respectively to 0.1 and 0.9 . The Goodput is improved by decreasing the number of neighbors of the active cognitive user $v$. The reason is obvious, more neighbors mean more active SUs which will arouse more collisions. For different values of $D e g_{v}$ there is a local maximum of the graph. For low values of $D e g_{v}$, the Goodput comes close to his maximum value.

Fig. 10 shows the achieved Goodput metric in terms of redundancy $X$ for different number of slots $M$. The $\operatorname{Deg}_{v}$ value has been fixed at 3 and the probabilities $p$ and $q$ has been fixed respectively to 0.1 and 0.9 . The proposed model exhibits good performance in terms of Goodput while decreasing the number of slots $M$ since fewer slots can be subject to eventual

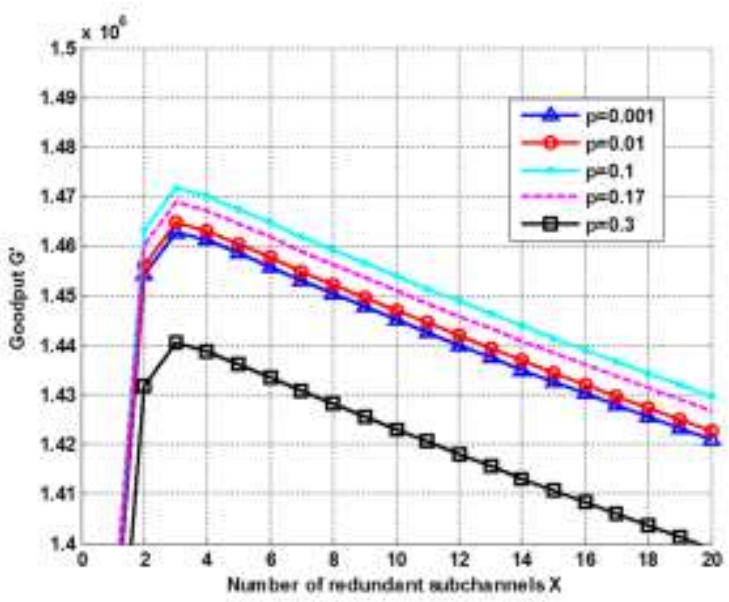

Figure 8. Goodput comparison for different values of the probability $p$ $(q=0.9)$

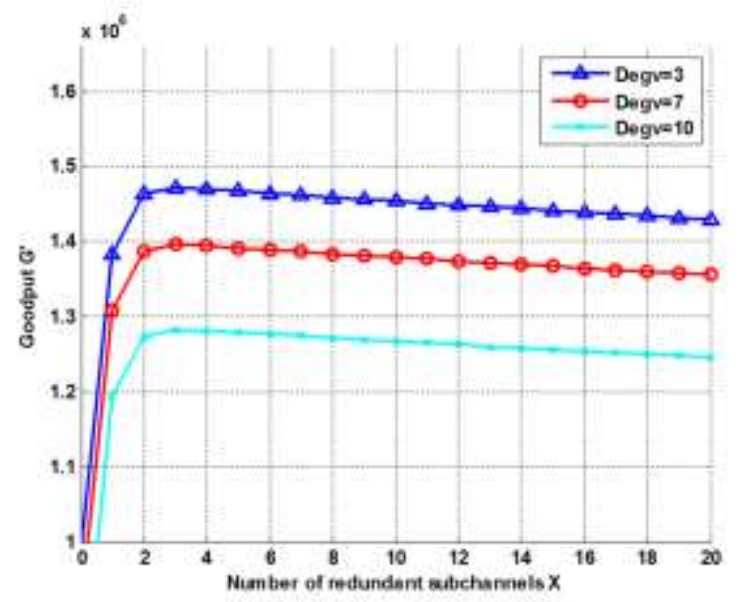

Figure 9. Achieved Goodput comparaison for different $\operatorname{Deg}_{y}$ values collisions. Increasing the number of slots $M$ enlarge the portions of time where different SUs could be active and collide with one another.

Fig. 11 illustrates the obtained Goodput in view of redundancy $X$ for several sought qualities. The $D e g_{v}$ value has been fixed at 3 and the probabilities $p$ and $q$ has been fixed respectively to 0.1 and 0.9 . Where increasing the number of transmitted packets, the achieved Goodput increases. Nevertheless, we notice that where increasing the packets number, the Goodput get away from his local maximum (see Fig. 11 and Tab. 1) which outlines the real need for reaching a good compromise between the computed Goodput and the expected quality.

\section{B. Analysis and discussions}

The proposed model has many parameters that influence the stability of the system such as, inter alia, the average traffic on the assigned slot, the average traffic on the remaining slots, frame size, neighbors' number, redundancy and number of available Secondary User Links. This is due to the fact that our model considers two critical features of the Cognitive Radio networks: Primary interruptions and Secondary Opportunistic Spectrum Sharing. The last factor was not taken into

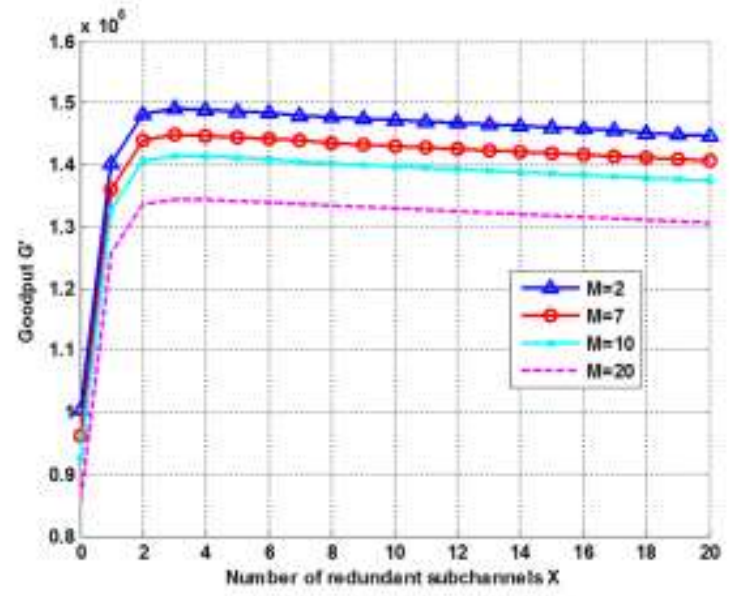

Figure 10. Achieved Goodput comparaison for different $M$ values 


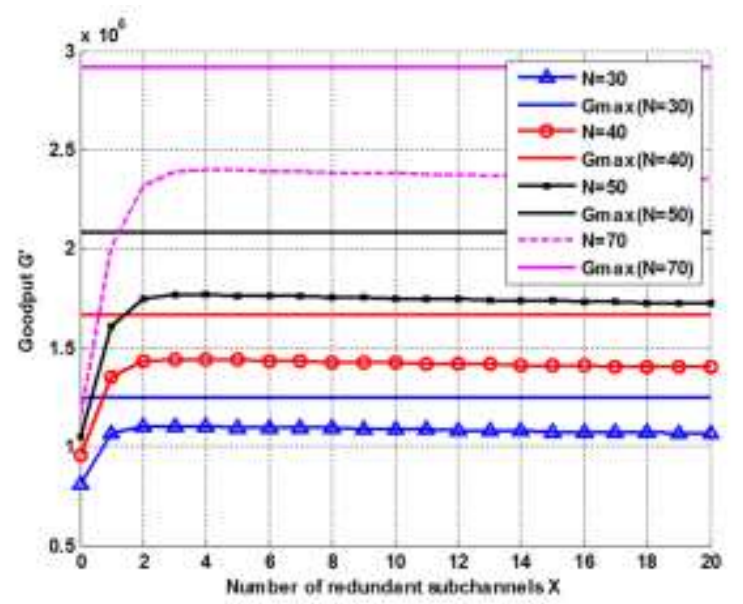

Figure 11. Achieved Goodput comparaison for different expected qualities consideration in [16] where the system is only sensitive to the amount of added redundancy. It is apparent that studying other Cognitive Radio factors will render the system stability more complex but also challenging. The system parameters need to be accordingly well adjusted.

The centralized scheduler set the value of slots number $M$, and the network architecture imposes the number of neighbors of each SU Deg $g_{s u}$. During the sensing phase, the CR system determines the vacant subchannels ready for secondary use; hence the Secondary Users Links $N_{S U L}$ could be established using the technique introduced in [15]. The average traffics $p$ and $q$ and the additional redundancy $X$ values could be analytically derived by addressing the Goodput maximization problem explored in the previous paragraph.

It is also shown that depending on the quality that we seek, we need to ensure a specific video data rate. Where our video transmission is "quality hungry", we must support higher bit rates and then our video transmission parameter settings have to be chosen adaptively depending on our system limitation. As a matter of fact, where increasing the transmission rate, we ameliorate the quality of the received video but more

TABLE I. QUALITIES AND MAXIMUM GOODPUTS

\begin{tabular}{|l|l|}
\hline \multicolumn{1}{|c|}{$\begin{array}{c}\text { Packets number (sought } \\
\text { quality) }\end{array}$} & \multicolumn{1}{|c|}{ Maximum Goodput (Mbit) } \\
\hline 30 & 1.25 \\
\hline 40 & 1.66 \\
\hline 50 & 2.08 \\
\hline 70 & 3 \\
\hline
\end{tabular}

subchannels are needed to successfully achieve this communication, however if we use less spectral resources, we optimize the use of the cognitive resources but the quality of the video stream at the consumer is degraded. We stat that when increasing the transmitted quality, it does not necessarily result in a more reliable transmission.

\section{CONCLUSION}

In this paper, we consider scalable video transmission over Cognitive Radio networks. The primary network has a binomial-modeled traffic. We have suggested making use of a progressive source coding associated to a fountain code. Then, we have evaluated the impact of the primary traffic interruptions on the secondary traffic and used a general model for collisions to modelize the opportunistic access of secondary users to CR network. Further, we have exploited a simple duplication-based mechanism for SULs to ameliorate the Goodput of the video transmission and make the SUs concurrency more infrequent. Our numerical results have been presented in terms of computed Goodput of the system. The achieved gain, while increasing the SULs number, proves the effectiveness of the given solution in terms of QoS requirements for video communication in secondary use. The paper concludes by emphasizing the importance of finding a balance that meets expected quality and achieved Goodput of the system. Hence, our video transmission parameters should be carefully chosen.

\section{REFERENCES}

[1] http://www.fcc.gov/oet/info/database/spectrum/

[2] Shared Spectrum Compagny. Spectrum occupancy measurement. site internet, http://www.sharedspectrum.com/measurements/.

[3] NTIA, "U.S. frequency allocations." [Online]. Available: http://www.ntia.doc.gov/osmhome/allochrt.pdf

[4] J. Mitola III, "Cognitive radio: an integrated agent architecture for software defined radio," Ph.D Thesis, KTH Royal Institute of Technology, 2000.

[5] FCC, ET Docket no. 03-322. Notice of Proposed Rule Making and Order, December 2003

[6] D. Cabric, S. M. Mishra, D. Willkomm, R. W. Broderson, and A. Wolisz, "A cognitive radio approach for usage of virtual unlicensed spectrum," in 14th IST Mobile Wireless Communications Summit 2005, Dresden, Germany, June 2005.

[7] I. Akyildiz, Y. Altunbasak, F. Fekri, and R. Sivakumar, "AdaptNet: an adaptive protocol suite for the next-generation wireless Internet," Communications Magazine, IEEE , vol.42, no.3, pp. 128- 136, Mar 2004.

[8] I. F. Akyildiz, W. Y. Lee, M. C. Vuran, and S. Mohanty, "Next generation/dynamic spectrum access/cognitive radio wireless networks: A survey," Computer Networks Journal, vol. 50, Sept. 2006.

[9] C. Cordeiro, K. Challapali, D. Birru, and N. Sai Shankar, "IEEE 802.22: the first worldwide wireless standard based on cognitive radios," IEEE DySPAN, pp.328-337, Nov. 2005.

[10] B. Ishibashi, N. Bouabdallah, and R. Boutaba, "QoS performance analysis of cognitive radio-based virtual wireless networks," INFOCOM 2008. The 27th Conference on Computer Communications. IEEE, vol., no., pp.2423-2431, 13-18 April 2008.

[11] H. Kushwaha, Y. Xing, R. Chandramouli, and H. Heffes, "Reliable multimedia transmission over cognitive radio networks using fountain codes," Proc. IEEE, vol. 96, no. 1, pp. 155-165, Jan. 2008.

[12] M. Luby, "LT codes," Proc. 43rd Ann. IEEE Symp. on Foundations of Computer Science, 2002, pp. 271-282.

[13] L. Cuiran and L. Chengshu, "Opportunistic spectrum access in cognitive radio networks," Neural Networks, 2008. IJCNN 2008. (IEEE World Congress on Computational Intelligence). IEEE International Joint Conference on , vol., no., pp.3412-3415, 1-8 June 2008. 
[14] A. Chaoub, E. Ibn Elhaj, and J. El Abbadi, "Unequal protected fountain code for progressive image source coding using block duplication," Seventh JFMMA \& TELECOM 2011, Tangier, Morocco, March 2011.

[15] A. Chaoub, E. Ibn Elhaj, and J. El Abbadi, "Multimedia traffic transmission over TDMA shared cognitive radio networks with poissonian primary traffic," Multimedia Computing and Systems, 2011. ICMCS '11. International Conference on, vol., no., pp.1-6, 7-9 April 2011.

[16] D. Willkomm, J. Gross, and A. Wolisz, "Reliable link maintenance in cognitive radio systems," in Proc. IEEE Symp. New Frontiers Dyn. Spectrum Access Netw. (DySPAN 2005), Baltimore, MD, Nov. 2005.

[17] A. Said and W. A. Pearlman, "A new, fast, and efficient image codec based on set partitioning in hierarchical trees," IEEE Trans. Circuits Syst. Video Technol., vol. 6, pp. 243-250, June 1996.

[18] D.J.C. MacKay, "Fountain codes," IEE Proc.-Commun., vol. 152(6), 2005, pp.1062-1068.

[19] A. Chaoub, E. Ibn Elhaj, and J. El Abbadi, "Multimedia traffic transmission over cognitive radio networks using multiple description coding," ACC 2011, Part I, CCIS 190, pp. 529-543, 2011, SpringerVerlag Berlin Heidelberg 2011.

[20] A. Shokrollahi, "Raptor codes," IEEE Trans. Inform. Theory, vol. 52, pp. 2551-2567, June 2006.
[21] T. Weiss and F. Jondral, "Spectrum pooling: an innovative strategy for the enhancement of spectrum efficiency," IEEE Communications Magazine, Vol. 42, no. 3, March 2004, pp. 8-14.

[22] R. W. Broderson, A. Wolisz, D. Cabric, S. M. Mishra, and D. Willkomm, "Corvus: a cognitive radio approach for usage of virtual unlicensed spectrum," White Paper, Univ. California Berkeley, Tech. Rep., Jul. 2004.

[23] H. Su and X. Zhang, "Cross-Layer Based Opportunistic MAC Protocols for QoS Provisionings Over Cognitive Radio Wireless Networks," IEEE Journal on Selected Areas in Communications (J-SAC), Vol. 26, No. 1, pp. 118-129, January 2008.

[24] J. Tang and X. Zhang, "Quality-of-service driven power and rate adaptation over wireless links," IEEE Transactions on Wireless Communications, Vol. 6, No. 8, pp. 3058-3068, August 2007.

[25] X. Zhang, J. Tang, H. H. Chen, S. Ci, and M. Guizani, "Cross-layer based modeling for quality of service guarantees in mobile wireless networks," IEEE Communications Magazine, Vol. 44, No. 1, pp. 100 106, January, 2006.

[26] J. Tang and X. Zhang, "Cross-layer-model based adaptive resource allocation for statistical QoS guarantees in mobile wireless networks," IEEE Transactions on Wireless Communications, Vol. 7, No. 6, pp. 2318-2328, June 2008.

[27] R. Koenen, "MPEG-4 multimedia for our time," IEEE Spectrum, vol. 36, no. 2, Feb. 1999, pp. 26-33. 